\title{
Soft landing of metal clusters on graphite: a molecular dynamics study *
}

\author{
Alexey V. Verkhovtsev ${ }^{1,3, a}$, Yury Erofeev ${ }^{1,2}$, and Andrey V. Solov'yov ${ }^{1,3}$ \\ 1 MBN Research Center, Altenhöferallee 3, 60438 Frankfurt am Main, Germany \\ 2 Department of Physics, Utrecht University, Heidelberglaan 8, 3584 CS Utrecht, The Netherlands \\ 3 On leave from Ioffe Institute, Polytekhnicheskaya 26, 194021 St. Petersburg, Russia
}

Received 7 May 2020 / Received in final form 24 August 2020 / Accepted 1 September 2020

Published online 6 October 2020

(C) EDP Sciences / Società Italiana di Fisica / Springer-Verlag GmbH Germany, part of Springer Nature, 2020

\begin{abstract}
Structure and stability of nanometer-sized $\mathrm{Ag}_{887}, \mathrm{Au}_{887}$ and $\mathrm{Ti}_{787}$ clusters soft-landed on graphite (at deposition energies $E_{\mathrm{dep}}=0.001-5.0 \mathrm{eV}$ per atom) are studied by means of molecular dynamics simulations. Parameters for the cluster-surface interactions are derived from complementary ab initio calculations. The shape and the contact angle of deposited clusters are systematically analyzed for different deposition energies and temperature regimes. The $\mathrm{Ag}_{887}$ cluster deposited at $E_{\text {dep }} \lesssim 0.1 \mathrm{eV} /$ atom undergoes collision-induced plastic deformation, thus acquiring an ellipsoidal shape with the contact angle close to $180^{\circ}$. In contrast, $\mathrm{Au}_{887}$ and $\mathrm{Ti}_{787}$ clusters undergo a collision-induced melting phase transition followed by their recrystallization; these processes lead to the formation of the droplet-like shapes of the clusters in a form of truncated spheroids. At larger deposition energies all clusters flatten over the surface and eventually disintegrate at $E_{\mathrm{dep}} \approx 0.75-1.0 \mathrm{eV} /$ atom (for $\mathrm{Ag}_{887}$ and $\mathrm{Au}_{887}$ ) and $\approx 3 \mathrm{eV} / \mathrm{atom}$ (for $\mathrm{Ti}_{787}$ ). It is found also that the shape of deposited clusters is strongly influenced by the strength of cluster-substrate interaction and the corresponding interaction mechanism, namely the weak van der Waals interaction between metal and carbon atoms or the van der Waals interaction with an onset of covalent bonding. Similar phenomena should arise in the deposition of clusters made of other elements, which interact with a substrate by one of the above-described mechanisms.
\end{abstract}

\section{Introduction}

The interaction of atomic clusters and nanoparticles with surfaces has been a widely studied topic in cluster science over the past several decades [1-3]. The dynamics of metal clusters and carbon fullerenes deposited onto different surfaces (mainly, metal surfaces, graphite and silicon oxide) was explored both experimentally [4-7] and computationally by means of molecular dynamics (MD) simulations [8-10]. The strong interest in the deposition of mass-selected clusters on surfaces has been motivated by both fundamental research and technological applications.

From a fundamental physics viewpoint, an important question is how structural, electronic, magnetic and optical properties of deposited clusters change with respect to free counterparts. A variety of phenomena emerge also when atomic clusters are brought in contact with a surface [11]. Examples include fragmentation of clusters and implantation of cluster atoms into the substrate [12],

* Contribution to the Topical Issue "Atomic Cluster Collisions (2019)", edited by Alexey Verkhovtsev, Pablo de Vera, Nigel J. Mason, Andrey V. Solov'yov.

a e-mail: verkhovtsev@mbnexplorer.com penetration of energetic clusters through a substrate and surface sputtering [13,14], irradiation-induced structural rearrangements of deposited clusters [15], cluster diffusion and aggregation into islands [16,17], super-diffusion and Lévy flights [18], as well as the formation and fragmentation of fractal nanostructures [19-21].

From a technological viewpoint, an understanding of the cluster-surface interaction is crucial for the controllable production of novel materials such as thin films and nanostructured surfaces [22], supported nanocatalysts [23] as well as nanoscale components for electronic devices [24]. If the process of cluster landing on a surface significantly modifies the shape and morphology of the clusters, the major technical effort required to produce size-selected cluster beams is largely in vain. Stability of size-selected clusters on a surface is therefore a key goal of deposition studies.

Stability and electronic properties of small clusters deposited on solid surfaces were also studied theoretically within the framework of the liquid drop model [25-27]. Analytical relations were derived for the deformationdependent surface and curvature energies of small $\mathrm{Na}_{N}$ and $\operatorname{Ar}_{N}(N<150)$ clusters of different shapes (oblate and prolate spheroids, semi-spheroids and truncated 
ellipsoids) deposited on a solid surface. Sequences of "magic" numbers for spheroidal and semi-spheroidal clusters were determined for different values of the deformation parameter [26]. These results demonstrated the important role of deformation and quantum effects in determining the stability of small atomic clusters on a substrate.

The wetting properties of solid surfaces have been intensively studied over the past decades. As such, many experimental and theoretical studies have been devoted to contact angle measurements of liquid droplets (e.g. water droplets or water-ethanol mixtures) [28-30] and liquid metal droplets $[31,32]$. However, to the best of our knowledge, the contact angle between a solid metal cluster and a surface has never been determined either experimentally or theoretically. A question of how the contact angle may be influenced by the cluster deposition conditions has not been analyzed either.

This paper reports a computational study of structure and stability of large ( $3 \mathrm{~nm}$ in diameter) silver, gold and titanium clusters, $\mathrm{Ag}_{887}, \mathrm{Au}_{887}$ and $\mathrm{Ti}_{787}$, deposited on graphite. We focused on the deposition energy range of 0.001-5.0 eV per atom, which corresponds to the softlanding regime utilized in numerous experiments (e.g., Refs. [19-21,33] to name a few). Other phenomena that arise at more energetic collisions, e.g. cluster pinning or the formation of holes due to penetration of clusters through a substrate [12-14], are beyond the scope of this study but can also be simulated using the computational approach described below. As far as we know, a comparative analysis of the dynamics of clusters made of different elements and deposited at the same conditions has been lacking in the previous theoretical studies, each of which focused on one type of atomic clusters.

The shape, contact angle, contact radius and height of the deposited clusters are systematically analyzed for different deposition energies $E_{\text {dep }}$ and temperature regimes. The dependence of these parameters on the initial structure and internal energy of the clusters is also elaborated. Thermally excited clusters have smaller contact angles with graphite as compared to their cold counterparts. The dependence of the contact angle on cluster temperature is, however, different for the three cluster types considered. There are small variations of the shape of $\mathrm{Ti}_{787}$ at cluster temperatures up to $900 \mathrm{~K}$ and deposition energies up to $0.25 \mathrm{eV} /$ atom, whereas the shape of thermally excited $\mathrm{Ag}_{887}$ and $\mathrm{Au}_{887}$ clusters changes significantly even at low deposition energies. At $E_{\text {dep }} \lesssim 0.1 \mathrm{eV} /$ atom, the $\mathrm{Ag}_{887}$ cluster acquires an ellipsoidal shape as a result of collision-induced plastic deformation. A large increase of the internal energy of $\mathrm{Au}_{887}$ and $\mathrm{Ti}_{787}$ clusters deposited at $E_{\text {dep }} \lesssim 0.1 \mathrm{eV} /$ atom leads to fast melting and recrystallization of the clusters, which happen over several tens of picoseconds. These processes lead to the formation of the droplet-like shapes of the clusters in a form of truncated spheroids. At larger deposition energies all clusters flatten over the surface and eventually disintegrate at $E_{\text {dep }} \approx 0.75-1.0 \mathrm{eV} /$ atom (for $\mathrm{Ag}_{887}$ and $\mathrm{Au}_{887}$ ) and $\approx 3 \mathrm{eV} /$ atom (for $\mathrm{Ti}_{787}$ ).

We found also that the shape of deposited clusters is strongly influenced by the strength of cluster-substrate interaction. Parameters for the interaction between metal ( $\mathrm{Ag}, \mathrm{Au}$ and $\mathrm{Ti}$ ) atoms and graphite were obtained from complementary ab initio calculations employing the second-order Møller-Plesset (MP2) perturbation theory. Three types of metal clusters considered in this study interact differently with graphite. Physisorption of the silver cluster on graphite occurs due to the weak van der Waals interaction between silver and carbon atoms. The interaction of the gold cluster with graphite is mediated by van der Waals forces with a small onset of covalent bonding between gold and carbon atoms. In contrast, a stronger titanium-graphite interaction stems from a $p-d$ hybridization of atomic orbitals of carbon and titanium atoms. We expect that similar phenomena shall arise in the deposition of metal clusters made of other elements (e.g., Pt, Co, Ni, Pd), which interact with a substrate by one of the above-described mechanisms.

\section{Computational methodology}

The simulations were performed using MBN Explorer [34] - a software package for advanced multiscale modeling of complex molecular structure and dynamics. MBN Studio [35], a dedicated graphical user interface for MBN Explorer, was used to construct the systems, prepare all necessary input files and analyze simulation outputs.

\subsection{Metal clusters}

As a first step, spherical clusters with the diameter of $3 \mathrm{~nm}$ were cut from ideal silver, gold and titanium crystals. The resulting structures contained $N_{\mathrm{Ag}}=N_{\mathrm{Au}}=887$ and $N_{\mathrm{Ti}}=787$ atoms. Energy minimization calculations were conducted for the free clusters using the velocity quenching algorithm [36] with the time step of 1 fs. Interatomic interactions were described using the many-body Gupta potential [37] and the parameters were taken from reference [38].

Energy-minimized structures were annealed by means of MD simulations following the computational protocol from reference [39]. The annealing procedure enabled sampling of the configuration space of the clusters at elevated temperatures, at which the cluster surface has undergone the melting phase transition but the core remained, at least partly, in the solid phase. This protocol was validated [39] by the comparison of annealed cluster geometries with the structures obtained from scanning transmission electron microscopy experiments. The clusters were heated up to $700 \mathrm{~K}$ for $\mathrm{Au}_{887}, 800 \mathrm{~K}$ for $\mathrm{Ag}_{887}$ and $900 \mathrm{~K}$ for $\mathrm{Ti}_{787}$. These values are about $70-100 \mathrm{~K}$ lower than the melting temperatures of the clusters which were determined through the analysis of caloric curves and rootmean-square displacement of all atoms. Each cluster was heated from $0 \mathrm{~K}$ up to the target temperature for $1 \mathrm{~ns}$, then kept at that temperature for 2 ns and cooled down to $0 \mathrm{~K}$ over $1 \mathrm{~ns}$. One annealing cycle was therefore completed in 4 ns. Three subsequent cycles were performed for each cluster to obtain energetically favorable structures. 

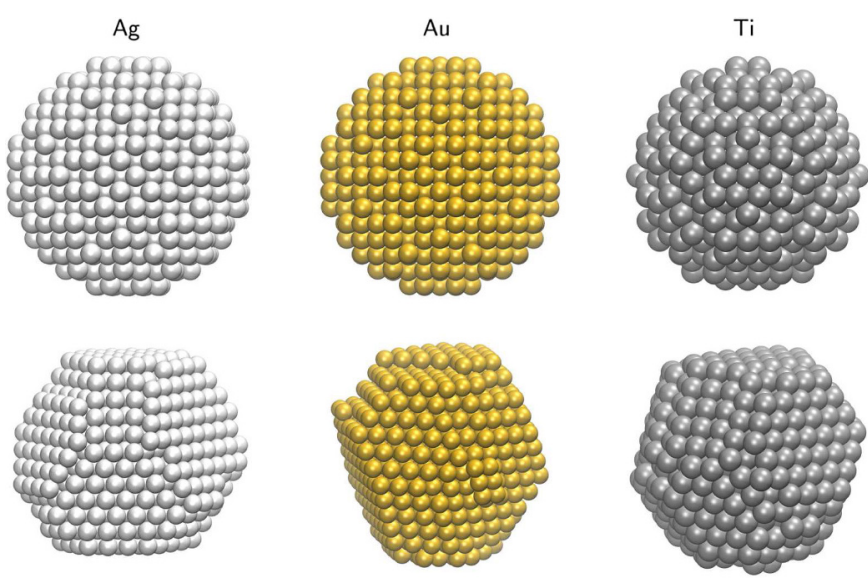

Fig. 1. Geometries of $\mathrm{Ag}_{887}, \mathrm{Au}_{887}$ and $\mathrm{Ti}_{787}$ clusters obtained after energy minimization (upper row) and the annealing process (bottom row).

Potential energy of each cluster decreased upon annealing by about $0.02 \mathrm{eV} /$ atom with respect to the initial values obtained from energy minimization. The annealed cluster structures contained several grains with different (mainly, fcc and hcp) crystal lattices. Final structures of the energy-minimized and annealed clusters are compared in Figure 1.

\subsection{Graphite substrate}

The constructed graphite substrate contained 5 monolayers with the size of $106.35 \times 104.38 \AA^{2}$. These dimensions were chosen to replicate the system with periodic boundary conditions. The substrate size was more than three times larger than the cluster size and significantly exceeded the range of interatomic interactions as described below. Thus, we ensured that there is no artificial interaction across the simulation box boundaries. Prior simulation of cluster deposition, the substrate was energy minimized using the velocity quenching algorithm. The many-body Brenner potential [40] was used to describe the interaction between covalentlybonded carbon atoms within each graphite layer whereas the Lennard-Jones potential was employed to account for the van der Waals interaction between the layers. The Lennard-Jones potential is implemented in MBN Explorer in the following form:

$$
U(r)=\varepsilon\left[\left(\frac{r_{0}}{r}\right)^{12}-2\left(\frac{r_{0}}{r}\right)^{6}\right],
$$

where $\varepsilon$ is the depth of the potential energy well and $r_{0}$ is the equilibrium interatomic distance. Parameters for the carbon-carbon interaction (see Tab. 1) were taken from reference [41]. The Lennard-Jones potential was truncated at a cutoff distance of $10 \AA$ that is about 3 times greater than the interplanar distance in graphite and an order of magnitude smaller than the size of the simulation box.
Table 1. Parameters of the Lennard-Jones potential used in this work to describe the $\mathrm{C}-\mathrm{C}$ interaction between graphite layers as well as between the metal $(\mathrm{Ag}, \mathrm{Au}, \mathrm{Ti})$ and carbon atoms. Other values (of the Lennard-Jones or the pairwise Morse potentials) reported in literature are also listed for comparison.

\begin{tabular}{lccc}
\hline & $\varepsilon(\mathrm{eV})$ & $r_{0}(\AA)$ & Reference \\
\hline $\mathrm{C}-\mathrm{C}$ & 0.00286 & 3.89 & {$[41]$} \\
$\mathrm{Ag}-\mathrm{C}$ & 0.020 & 3.49 & This work (MP2) \\
& 0.029 & 3.32 & {$[42]$} \\
& 0.030 & 3.37 & {$[43]$} \\
& 0.009 & 3.45 & {$[46]$ (Morse) } \\
$\mathrm{Au}-\mathrm{C}$ & 0.044 & 3.49 & This work (MP2) \\
& 0.033 & 3.32 & {$[42]$} \\
& 0.013 & 3.36 & {$[18]$} \\
$\mathrm{Ti}-\mathrm{C}$ & 0.165 & 2.44 & This work (MP2) \\
$\mathrm{Ni}-\mathrm{C}$ & 0.023 & 3.20 & {$[42]$} \\
& 0.345 & 2.03 & {$[45]$ (Morse) } \\
& 0.363 & 2.28 & {$[46]$ (Morse) } \\
\hline
\end{tabular}

\subsection{Metal-carbon interaction}

The cluster-substrate interaction was also described by the Lennard-Jones potential using the cutoff distance of $10 \AA$.

Several MD studies of noble and transition-metal clusters $(\mathrm{Ag}, \mathrm{Au}, \mathrm{Pt}, \mathrm{Cu}, \mathrm{Ni})$ interacting with graphite and graphene were reported earlier, see e.g. references $[18,42$, 43]. Parameters for the metal-carbon (M-C) interaction were derived in those papers using empirical mixing rules:

$$
r_{0}^{\mathrm{M}-\mathrm{C}}=\frac{1}{2}\left(r_{0}^{\mathrm{M}-\mathrm{M}}+r_{0}^{\mathrm{C}-\mathrm{C}}\right), \quad \varepsilon^{\mathrm{M}-\mathrm{C}}=\sqrt{\varepsilon^{\mathrm{M}-\mathrm{M}} \varepsilon^{\mathrm{C}-\mathrm{C}}},
$$

where $r_{0}^{\mathrm{M}-\mathrm{M}}$ and $\varepsilon^{\mathrm{M}-\mathrm{M}}$ are the parameters of metal-metal interactions [44]. Other studies have shown $[45,46]$ that parameters for the pairwise interactions between metal atoms and $s p^{2}$ carbon systems, such as fullerenes or carbon nanotubes, differ significantly from those derived using the mixing rules. Thus, a broad range of parameters for various metal-carbon systems can be found in literature, and the optimal choice of the parameters is not obvious.

To elaborate on this issue, we performed ab initio calculations of potential energy scans for $\mathrm{Ag}, \mathrm{Au}$ and $\mathrm{Ti}$ atoms interacting with a benzene molecule. Benzene as well as larger polycyclic aromatic hydrocarbon (PAH) molecules may be considered as prototypic structural units of graphene with their dangling bonds saturated with hydrogen atoms. Although a more accurate description of metal-graphene interaction is achieved as the number of carbon atoms in prototypic molecules increases up to $\sim 10^{2}[47,48]$, ab initio calculations of such large PAH molecules are challenging from a computational point of view. It is known (see, e.g., Refs. [49,50] and Refs. therein) that standard DFT methods do not account properly for long-range dispersion interactions and require additional empirical corrections. The importance of non-local correlation effects that govern dispersive interactions was 
particularly emphasized for the binding of atoms and small clusters of silver and gold on graphite [51,52].

Since the dispersive interaction is naturally accounted for in wave function-based ab initio methods, a series of calculations employing the second-order Møller-Plesset (MP2) perturbation theory were performed. The Gaussian 09 software package [53] using a LanL2DZ basis set was employed. The metal atoms were placed in the hollow position on top of the benzene molecule and displaced along its main axis. The metal atom-benzene interaction energy was obtained from these scans and divided by the number of carbon atoms to determine the interaction energy per an $\mathrm{M}-\mathrm{C}$ pair of atoms. Since benzene is a highly symmetric molecule and the metal atoms were displaced along its main axis, all $\mathrm{M}-\mathrm{C}$ bonds can be considered as equivalent. The evaluated parameters of the Lennard-Jones potential for $\mathrm{Ag}-\mathrm{C}, \mathrm{Au}-\mathrm{C}$ and $\mathrm{Ti}-\mathrm{C}$ are summarized in Table 1. Other values reported in literature $[18,42,43,46]$ are also presented for completeness. Note that the parameters from references $[18,42,43]$ were determined using the mixing rules (Eq. (2)) while the parameters from references $[45,46]$ were derived on the basis of DFT calculations. To the best of our knowledge, there are no reference data available on the interaction between $\mathrm{Ti}$ and $\mathrm{C}$ atoms. Therefore, in Table 1 we provide also parameters taken from literature $[42,45,46]$ for the interaction between carbon and nickel, another open-shell transition metal.

Our MP2 calculations revealed that the metal-carbon interaction energy increases from silver to gold to titanium. The $\mathrm{Au}-\mathrm{C}$ potential well depth is about two times larger than for the $\mathrm{Ag}-\mathrm{C}$ interaction. This is in agreement with the results of earlier DFT calculations with dispersion corrections [51] which found that adsorption energy of a gold atom on graphite is about two times higher than for a silver atom. It was also found [51] that no charge redistribution occurs between the Ag atom and graphite, while the deposited $\mathrm{Au}$ atom receives a charge of approximately $0.1 e$ from the carbon sheet. On this basis, it was concluded that the adsorption of silver on graphite is purely of van der Waals type whereas small hybridization in the density of states, i.e. a chemical contribution to the binding, occurs in the case of gold adsorbed on graphite. As it is indicated in Table 1 , the $\mathrm{Ti}-\mathrm{C}$ interaction is about eight times stronger than the $\mathrm{Ag}-\mathrm{C}$ interaction. A recent DFT-based study of the titanium-graphite interface [54] reported the formation of chemical bonding between interfacial $\mathrm{Ti}$ and $\mathrm{C}$ atoms. It was found also that each interfacial Ti atom acquires a charge of about $0.3 e$. Another DFT study of the adsorption of a titanium slab on graphene demonstrated that a $p-d$ hybridization occurs between atomic orbitals of carbon and titanium [55].

\subsection{Deposition of clusters on graphite}

A simulation box of $106.35 \times 104.38 \times 110 \AA^{3}$ was used for MD simulations of cluster deposition. Each cluster was placed in the center of the simulation box approximately $40 \AA$ above the topmost graphite layer. The clusters were deposited with energies $E_{\text {dep }}=$ $0.001,0.01,0.05,0.1,0.25,0.75$ and $1.0 \mathrm{eV} /$ atom at nor- mal incidence to the graphite surface. In order to determine fragmentation threshold for the $\mathrm{Ti}_{787}$ cluster, higher deposition energies from 2.0 to $5.0 \mathrm{eV} /$ atom were also considered.

To simulate the deposition of clusters and their rearrangement on a surface, 250-ps long MD simulations were performed for the microcanonical $(N V E)$ ensemble of particles. The simulation time was chosen such that, after hitting the surface, the clusters would relax for at least 200 ps. Kinetic and potential energies of the system reached steady-state values within $20-40$ ps after the collision and remained nearly constant until the end of each simulation. Integration of equations of motion was done using the velocity Verlet algorithm with the time step of $1 \mathrm{fs}$. This value was chosen to ensure that a variation of the total energy of the system did not exceed $0.01 \%$. Two bottom layers of graphite were fixed to avoid translational motion of the whole system after the collision. Test calculations were performed also for thicker graphite substrates containing 7 and 11 monolayers to check that the results obtained do not depend on substrate thickness.

As described in Section 3, disintegration of the clusters and scattering of cluster fragments over the whole graphite sample were observed at deposition energies of $0.75 \mathrm{eV} /$ atom and above. To ensure that the simulation box boundaries do not affect the results, a set of simulations was performed on a larger graphite substrate with the size of $212.7 \times 208.8 \AA^{2}$. The total number of atoms in the systems thus varied from approx. 19000 to 86000 .

\section{Results and discussion}

We begin our analysis by considering deposition of the spherical $\mathrm{Ag}_{887}, \mathrm{Au}_{887}$ and $\mathrm{Ti}_{787}$ clusters cut from ideal bulk crystals. Then we discuss how alteration of the initial cluster structure due to annealing affects their shape upon deposition. Finally, we consider deposition of thermally excited clusters and compare the resulting shapes with the case of deposition at zero temperature.

\subsection{Shape of deposited clusters}

For cluster sizes smaller than we considered in this study, quantum effects such as even-odd oscillations in cluster abundance spectra [56] and the appearance of "magic" numbers associated with electron shell closure [57], become more prominent and play a crucial role in determining the shape of clusters on a surface [26]. Such effects are particularly strong for the clusters containing $N \lesssim 200$ atoms but shrink with increasing the cluster size up to $N \approx 800$ [58].

Figure 2 shows final snapshots of the spherical $\mathrm{Ag}_{887}$, $\mathrm{Au}_{887}$ and $\mathrm{Ti}_{787}$ clusters deposited on graphite at different deposition energies $E_{\text {dep }}$. The figure indicates clearly that the cluster geometry depends on the element type and that it changes significantly with an increase of deposition energy. At $E_{\text {dep }}=0.001 \mathrm{eV} /$ atom (upper row) the $\mathrm{Ag}_{887}$ cluster retains its spherical shape, whereas the gold 


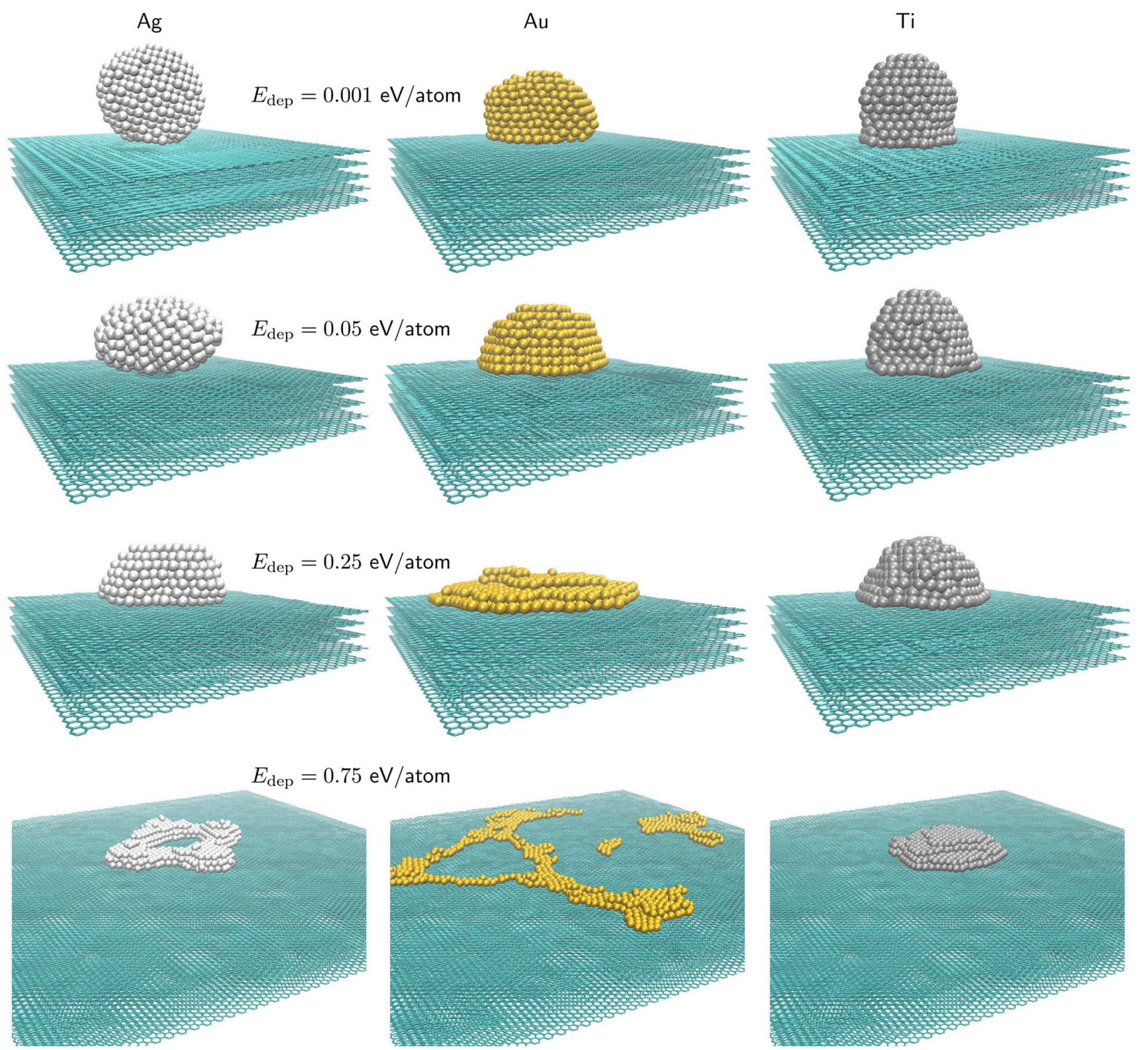

Fig. 2. MD snapshots of the spherical $\mathrm{Ag}_{887}$ (left column), $\mathrm{Au}_{887}$ (middle column) and $\mathrm{Ti}_{787}$ (right column) clusters deposited on graphite at the deposition energies of $0.001,0.05,0.25$ and $0.75 \mathrm{eV} /$ atom (top to bottom rows).

and titanium clusters are deformed due to stronger adhesion to the surface. The $\mathrm{Au}_{887}$ cluster has the shape of a truncated ellipsoid while $\mathrm{Ti}_{787}$ is a capped structure elongated in the direction normal to the substrate. At $E_{\text {dep }}=$ $0.05 \mathrm{eV} /$ atom (second row) the silver cluster acquires a slightly deformed quasi-ellipsoidal shape, while both gold and titanium clusters transform into truncated ellipsoids. All three clusters deposited at $E_{\text {dep }}=0.25 \mathrm{eV} /$ atom (third row) transform into truncated ellipsoids with the gold cluster being the most flattened structure. Finally, at $E_{\mathrm{dep}}=0.75 \mathrm{eV} /$ atom (bottom row) topology of the silver and gold clusters changes after collision with the surface. The $\mathrm{Ag}_{887}$ cluster becomes a hollow structure that remains stable over the 250 -ps long simulation. The $\mathrm{Au}_{887}$ cluster fragments into a large pretzel-like structure and several smaller islands that are scattered over the surface. In contrast, $\mathrm{Ti}_{787}$ remains intact but gradually becomes more and more flattened. Note that the simulations performed at $E_{\text {dep }}=0.75 \mathrm{eV} /$ atom were conducted on a large graphite substrate of $212.7 \times 208.8 \AA^{2}$.

Figure 3 shows the change of the internal energy of the $\mathrm{Ag}_{887}, \mathrm{Au}_{887}$ and $\mathrm{Ti}_{787}$ clusters,

$$
\Delta E^{\mathrm{cl}}=E^{\mathrm{cl}}(t)-E^{\mathrm{cl}}(t=0)
$$

as a function of simulation time $t \cdot E^{\mathrm{cl}}(t=0)$ denotes the potential energy of a free cluster. When the clusters hit the surface, $\Delta E^{\mathrm{cl}}$ increases sharply as the clusters experience both elastic and plastic deformations [59]. During the follow-up relaxation process, the internal energy of each 

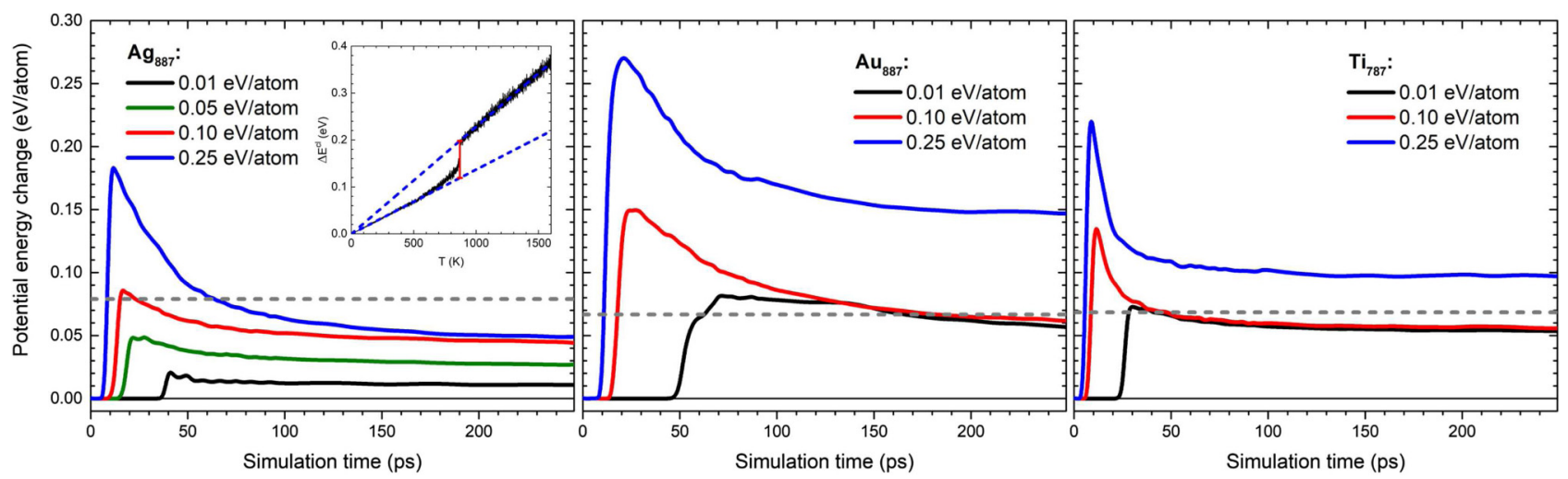

Fig. 3. The change of the internal energy of the $\mathrm{Ag}_{887}$ (left panel), $\mathrm{Au}_{887}$ (middle panel) and $\mathrm{Ti}_{787}$ (right panel) clusters, $\Delta E^{\mathrm{cl}}$, equation (3), deposited at different values of $E_{\mathrm{dep}}$, as a function of simulation time. Horizontal dashed lines show the internal energy change arising in the process of melting of free $\mathrm{Ag}_{887}, \mathrm{Au}_{887}$ and $\mathrm{Ti}_{787}$ clusters. A simulated caloric curve (a change of the internal energy as a function of temperature) for a free $\mathrm{Ag}_{887}$ is shown in the inset.

cluster decreases and asymptotically approaches a constant value which arises due to irreversible plastic deformations of the clusters.

Note that $\Delta E^{\mathrm{cl}}$ for $\mathrm{Ag}_{887}$ is much smaller than for the gold and titanium clusters deposited at a given energy $E_{\mathrm{dep}}$. The change of the internal energy of the silver cluster deposited at $E_{\mathrm{dep}}=0.01$ and $0.05 \mathrm{eV} /$ atom is significantly smaller than the change of the internal energy occurring during melting of the free $\mathrm{Ag}_{887}$ cluster (see the horizontal dashed line and the inset in the left panel of Fig. 3). This means that the $\mathrm{Ag}_{887}$ cluster deposited at small values of $E_{\text {dep }}$ undergoes collision-induced plastic deformation but does not melt in the course of simulation. This structural transformation governs an ellipsoidal shape of $\mathrm{Ag}_{887}$ deposited at $E_{\text {dep }}=0.05 \mathrm{eV} /$ atom.

In contrast, the internal energies of the $\mathrm{Au}_{887}$ and $\mathrm{Ti}_{787}$ clusters deposited at $E_{\text {dep }}=0.01 \mathrm{eV} /$ atom are comparable to the internal energy changes arising in the process of cluster melting (see dashed lines). This means that the gold and titanium clusters experience the melting phase transition upon collision with graphite, which is followed by recrystallization of the clusters. As a result, the $\mathrm{Au}_{887}$ and $\mathrm{Ti}_{787}$ clusters acquire the droplet-like shapes in a form of truncated spheroids as illustrated in Figure 2.

\subsection{Contact angle and deformation parameter of the clusters}

Coordinates of cluster atoms, extracted from every simulated MD trajectory, were used to parameterize the cluster shape and to evaluate a contact angle with the substrate. As follows from the simulated trajectories, each deposited cluster is, to a good approximation, radially symmetric with respect to its main axis. Thus, we introduced cylindrical coordinates $\rho$ and $z$, where

$$
\rho=\sqrt{\left(x-x_{\mathrm{CM}}\right)^{2}+\left(y-y_{\mathrm{CM}}\right)^{2}},
$$

with $x_{\mathrm{CM}}$ and $y_{\mathrm{CM}}$ being $x$ - and $y$-projections of the center of mass of each cluster. The $\rho$-axis lies in the graphite surface plane, whereas $z$-axis is perpendicular to the surface and $z=0$ corresponds to the average position of the topmost graphite layer. Figure 4 shows by symbols $(\rho, z)$ projections of all atoms in the $\mathrm{Ag}_{887}, \mathrm{Au}_{887}$ and $\mathrm{Ti}_{787}$ clusters deposited at $0.01 \mathrm{eV} /$ atom. Figure $4 \mathrm{a}$ shows that at low values of $E_{\text {dep }}$ the $\mathrm{Ag}_{887}$ cluster acquires the shape of a non-truncated ellipsoid which is slightly deformed from the side being in contact with graphite. The initially spherical $\mathrm{Au}_{887}$ cluster transforms into a truncated oblate spheroid (Fig. 4b) while the $\mathrm{Ti}_{787}$ cluster has a well pronounced prolate shape, i.e. its height is larger than the contact radius (Fig. 4c). Interestingly, atoms in the titanium cluster arrange into layers oriented parallel to graphite planes, that is different from the atomic arrangement in the silver and gold clusters. This can be explained by a stronger interaction between titanium and carbon atoms as compared to the $\mathrm{Ag}-\mathrm{C}$ and $\mathrm{Au}-\mathrm{C}$ interactions.

For each cluster we selected coordinates of atoms located on the surface and fitted the resulting profiles with the following surface equation $[60,61]$ :

$$
\rho(z)= \begin{cases}\sqrt{a\left(z-z_{0}\right)^{2}+b\left(z-z_{0}\right)+c}, & z \geq 0 \\ 0 & , \quad z<0\end{cases}
$$

where $a, b, c$ and $z_{0}$ are fitting parameters. This expression enables a description of different cluster shapes with a single fitting function without any geometrical assumptions on the cluster shape. From the least-squares fit of cluster profiles with equation (5) we determined the contact radius and height of each cluster as well as the contact angle with the substrate as functions of deposition energy. An inverse dependence $z(\rho)$ is shown by red solid curves in Figure 4.

The contact angle $\theta$ was evaluated by calculating the derivative of $z(\rho)$ at the point $\rho^{\prime}$ which corresponds to the average position of the bottom-most atomic layer of the clusters, $z^{\prime}=z\left(\rho^{\prime}\right)$, see the dashed lines in Figure 4. An expression for the contact angle is then given by [60]

$$
\theta=\arctan \left(\left.\frac{d z}{d \rho}\right|_{\rho=\rho^{\prime}}\right)=\frac{\pi}{2}+\arctan \left(\left.\frac{d \rho}{d z}\right|_{z=z^{\prime}}\right) .
$$



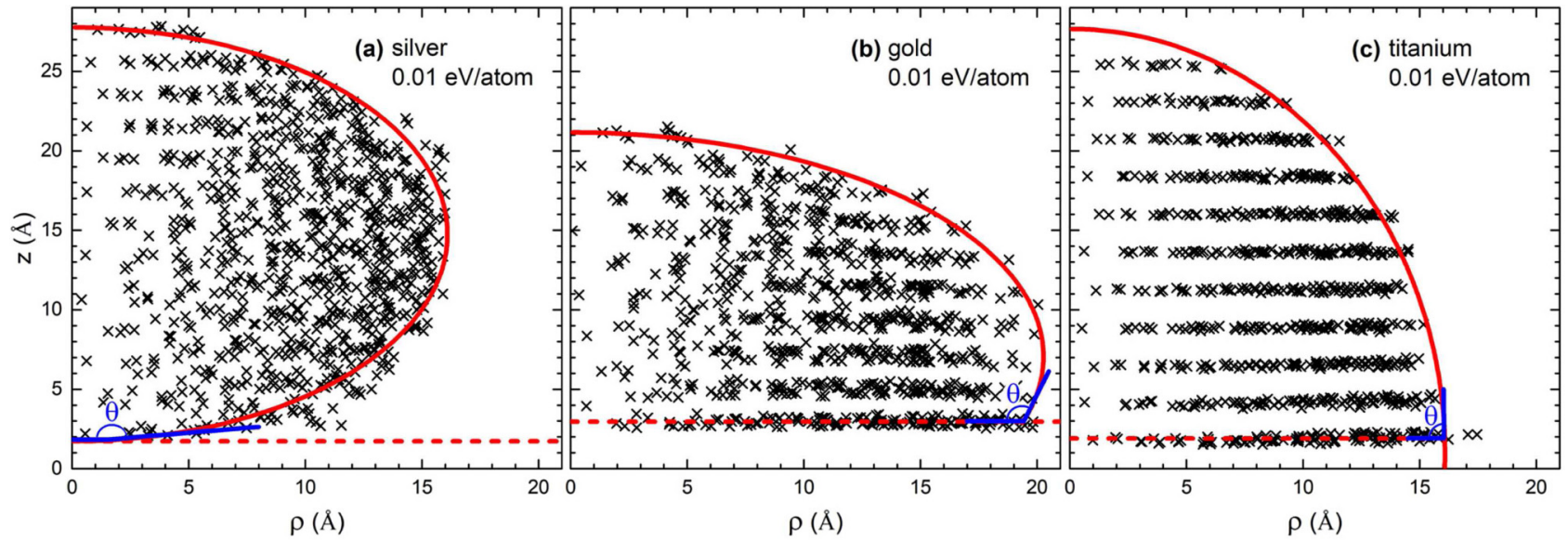

Fig. 4. Radial profiles of the initially spherical $\mathrm{Ag}_{887}$ (a), $\mathrm{Au}_{887}$ (b) and $\mathrm{Ti}_{787}$ (c) clusters deposited at $E_{\text {dep }}=0.01$ eV/atom. Symbols (crosses) show the distribution of all atoms in each cluster at the end of a 250 ps-long simulation. Solid curves show the best fit of the cluster profile with $z(\rho)$ that is an inverse function of $\rho(z)$, equation (5). Dashed lines indicate the averaged position of the bottom-most atomic layer in each cluster.

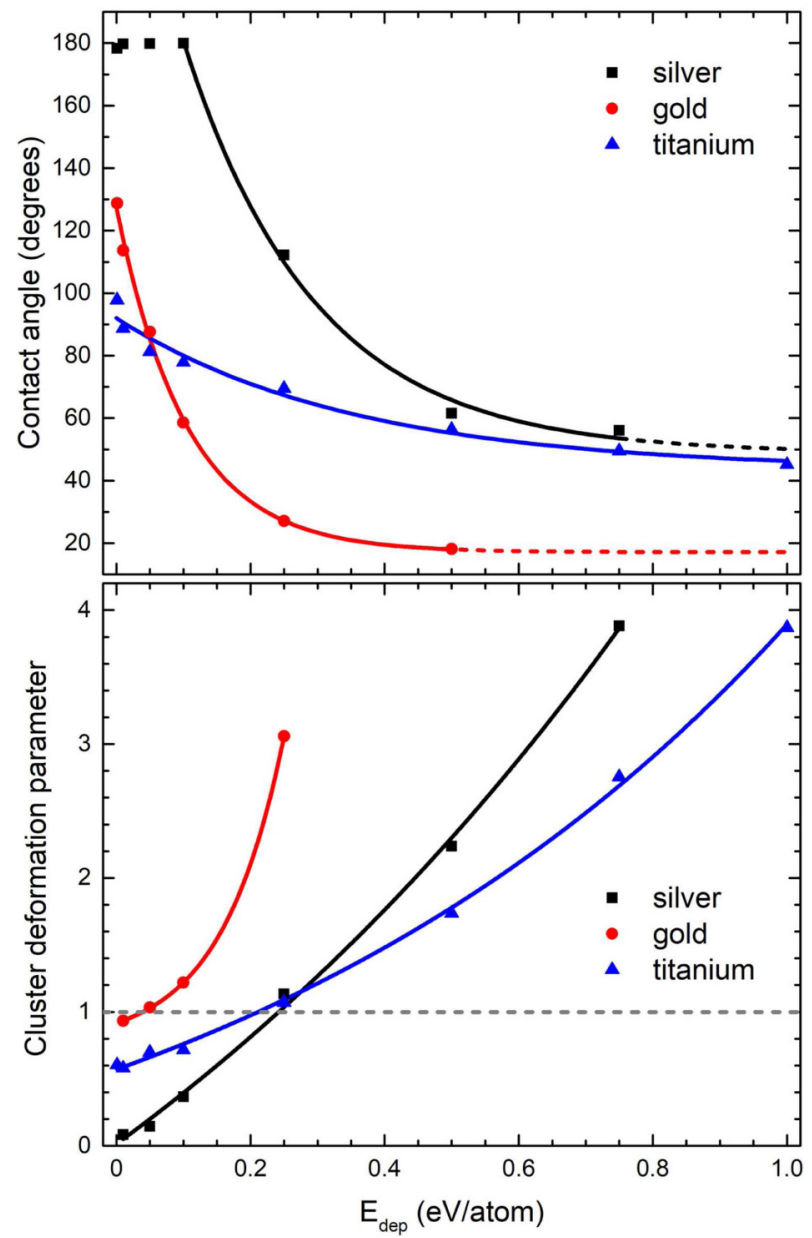

Fig. 5. Dependence of the contact angle (Eq. (6), top panel) and cluster deformation parameter (Eq. (8), bottom panel) on deposition energy for the initially spherical $\mathrm{Ag}_{887}, \mathrm{Au}_{887}$ and $\mathrm{Ti}_{787}$ clusters. Solid curves show exponential fits using equations (7) and (9).
The contact angle for the optimized $\mathrm{Ag}_{887}, \mathrm{Au}_{887}$ and $\mathrm{Ti}_{787}$ clusters as a function of deposition energy is presented in the upper panel of Figure 5. The calculated values of $\theta$ were averaged over three distinct cluster geometries sampled from the last $100 \mathrm{ps}$ of each simulation. It was found that the deposited clusters acquire their equilibrium shapes shortly after reaching the surface (on the timescales of several tens of picoseconds) and a variation of $\theta$ in the remaining part of simulations does not exceed 10 degrees.

The figure shows that the contact angle for the $\mathrm{Ag}_{887}$ cluster (black squares) evolves differently with an increase of $E_{\text {dep }}$ than for the gold and titanium clusters. The main distinction is that $\theta$ for $\mathrm{Ag}_{887}$ remains close to $180^{\circ}$ at deposition energies up to $0.1 \mathrm{eV} /$ atom. A further increase of $E_{\text {dep }}$ up to $0.25 \mathrm{eV} /$ atom leads to a rapid decrease of the angle by about 70 degrees whereas it converges to a value of about $60^{\circ}$ at $E_{\text {dep }}=0.75 \mathrm{eV} /$ atom. As discussed in Section 3.1, the $\mathrm{Ag}_{887}$ cluster deposited at $E_{\text {dep }} \lesssim 0.1 \mathrm{eV} /$ atom undergoes plastic deformation which is not followed by the melting phase transition. The rapid decrease of the contact angle at larger values of $E_{\text {dep }}$ occurs due to the melting and recrystallization of the cluster in the course of the deposition. At higher deposition energies, the cluster fragments into several small islands and therefore the contact angle was not determined. A similar trend was observed for $\mathrm{Au}_{887}$ (red circles). In this case, the contact angle drops rapidly from approx. $130^{\circ}$ at $E_{\text {dep }}=0.001 \mathrm{eV} /$ atom down to $20^{\circ}$ at $E_{\text {dep }}=0.5 \mathrm{eV} /$ atom. In the latter case, the cluster is rearranged into two atomic layers which are distributed uniformly over large graphite area. Deposition at higher energies also leads to cluster fragmentation and the formation of smaller islands with the height of two atomic layers. For the $\mathrm{Ti}_{787}$ cluster (blue triangles), $\theta$ gradually decreases from $100^{\circ}$ to $45^{\circ}$ in the deposition energy range considered. This corresponds to the observations shown in Figure 2 that the contact area for $\mathrm{Ti}_{787}$ increases 
Table 2. Fitting parameters describing an exponential decrease of the contact angle (Eq. (7)) and an increase of the cluster deformation parameter (Eq. (9)) with $E_{\text {dep }}$. Note that only the data points for $E_{\mathrm{dep}} \geq 0.1 \mathrm{eV} /$ atom were fitted with equation (7) for the $\mathrm{Ag}_{887}$ cluster.

\begin{tabular}{lccc}
\hline & $\mathrm{Ag}_{887}$ & $\mathrm{Au}_{887}$ & $\mathrm{Ti}_{787}$ \\
\hline$\theta_{0}$ (deg.) & 48.87 & 17.11 & 43.47 \\
$\theta_{1}$ (deg.) & 219.93 & 110.30 & 48.52 \\
$\alpha$ (eV ${ }^{-1} /$ atom $)$ & 5.13 & 9.58 & 2.83 \\
$\delta_{0}$ & -4.29 & 0.76 & -1.04 \\
$\delta_{1}$ & 4.30 & 0.16 & 1.61 \\
$\beta\left(\mathrm{eV}^{-1} /\right.$ atom $)$ & 0.85 & 10.74 & 1.12 \\
\hline
\end{tabular}

gradually with $E_{\text {dep }}$. Interestingly, the contact angle for all three clusters considered decreases exponentially with $E_{\text {dep }}$

$$
\theta=\theta_{0}+\theta_{1} e^{-\alpha E_{\mathrm{dep}}},
$$

see solid curves in the upper panel of Figure 5. The corresponding fitting parameters are summarized in Table 2.

Additional MD simulations were conducted at higher deposition energies to determine the fragmentation threshold for $\mathrm{Ti}_{787}$. We found that the threshold deposition energy is between 2 and $3 \mathrm{eV}$ per atom, which is about four times larger than the corresponding fragmentation thresholds for $\mathrm{Ag}_{887}$ and $\mathrm{Au}_{887}$. At $E_{\text {dep }}=2 \mathrm{eV} /$ atom the titanium cluster is flattened over the graphite surface but remains intact, whereas at $E_{\text {dep }}=3 \mathrm{eV} /$ atom the cluster transforms into a flat pretzel-like structure (similar to the $\mathrm{Au}_{887}$ cluster deposited at $0.75 \mathrm{eV} /$ atom, see Fig. 2) with the maximal height of two atomic layers. Deposition of $\mathrm{Ti}_{787}$ at $4 \mathrm{eV} /$ atom results in the formation of small titanium islands that are scattered over the whole simulated substrate. Note that the fragmentation thresholds should depend not only on elemental composition of the clusters but also on their size. However, we leave a detailed analysis of this dependence for further studies.

To complement this analysis, the lower panel of Figure 5 shows the cluster deformation parameter $\delta$, defined as a ratio of cluster contact radius (that is, radius of the bottom-most atomic layer, see Fig. 4) to cluster height,

$$
\delta=\frac{\rho\left(z=z^{\prime}\right)}{z(\rho=0)}
$$

for the initially spherical $\mathrm{Ag}_{887}, \mathrm{Au}_{887}$ and $\mathrm{Ti}_{787}$ clusters deposited at different energies. The dependence of the deformation parameter on $E_{\text {dep }}$ also follows an exponential law,

$$
\delta=\delta_{0}+\delta_{1} e^{\beta E_{\mathrm{dep}}},
$$

see solid curves in the lower panel of Figure 5. The corresponding fitting parameters are also listed in Table 2 . The horizontal dashed line denotes the case when the contact radius is equal to the height, which corresponds to a perfect semi-spheroidal shape. The figure shows that the shape of $\mathrm{Au}_{887}$ is close to a semi-spheroid at low deposition energies (up to $0.05 \mathrm{eV} /$ atom) while highly oblate truncated spheroids are formed in the course of the deposition at higher energies. A similar trend is observed for the silver and titanium clusters but in these cases the shape of the clusters is close to a semi-spheroid at $E_{\text {dep }}=0.25 \mathrm{eV} /$ atom. Thus, by increasing the deposition energy the clusters evolve from an ellipsoid (in the case of silver) or a truncated prolate spheroid (in the case of titanium) to semi-spheroids to very flat structures with the contact radius exceeding the cluster height by the factor of four.

The height and diameter of mass-selected clusters deposited on solid surfaces can be determined from atomic force microscopy and scanning tunnelling microscopy measurements. So far, the experimental data on the cluster height and size have been reported only in a few publications, e.g. [62,63]. In reference [62], the height of $\mathrm{Au}_{2000}$ clusters soft-landed on highly oriented graphite was determined as $3.2 \pm 0.4 \mathrm{~nm}$. The diameter of free $\mathrm{Au}_{2000}$ clusters is approx. $4 \mathrm{~nm}$ but no information was given in the cited paper on the diameter of deposited clusters. In reference [63], the height and diameter of mass-selected silver $\mathrm{Ag}_{2700}$ clusters soft-landed (at $0.25 \mathrm{eV} /$ atom) on graphite were determined as $4.1 \pm 0.3 \mathrm{~nm}$ and $6.2 \pm 2.5 \mathrm{~nm}$, respectively. Based on the reported experimental numbers, the deformation parameter for $\mathrm{Ag}_{2700}$ cluster can be evaluated as $0.8 \pm 0.3$. This value is in a good agreement with the value 1.1 obtained by us in MD simulations for the $\mathrm{Ag}_{887}$ cluster deposited at $E_{\text {dep }}=0.25 \mathrm{eV} /$ atom, see the bottom panel of Figure 5.

\subsection{Deposition of thermally excited clusters}

The above described analysis was carried out for the spherical clusters deposited at zero temperature. It is worth exploring how the shape of deposited clusters changes upon altering the initial cluster structure due to annealing. Results of this analysis are summarized in Figures 6 and 7, which show how the contact angle $\theta$ (Eq. (6)) and the deformation parameter $\delta$ (Eq. (8)) evolve as functions of $E_{\mathrm{dep}}$. Gray lines/open symbols correspond to the spherical cluster case described above in Figure 5. Black lines/filled squared represent the results for the annealed clusters (see Fig. 1) at initial temperature of $0 \mathrm{~K}$. The figures show that alteration of the cluster structure due to annealing has a moderate impact on the shape of $\mathrm{Ag}_{887}$ and $\mathrm{Ti}_{787}$ clusters in the deposition energy range considered, whereas annealing of $\mathrm{Au}_{887}$ results in a very different geometry even at low deposition energy. At $E_{\mathrm{dep}}=0.01 \mathrm{eV} /$ atom, the annealed gold cluster wets the surface stronger than its spherical counterpart and the contact angle decreases from $130^{\circ}$ down to $70^{\circ}$. However, the variation of $\theta$ with $E_{\text {dep }}$ is much smaller than for the spherical $\mathrm{Au}_{887}$ cluster so that at $E_{\text {dep }}=0.25 \mathrm{eV} /$ atom the contact angle for the annealed $\mathrm{Au}_{887}$ is almost two times larger than for the spherical cluster. Deposition of the annealed cluster at higher energies results in its fragmentation. Figure 7 indicates that the annealed noble metal clusters are characterized by larger values of the deformation parameter as compared to the spherical clusters but the annealing of a $\mathrm{Ti}_{787}$ cluster has a minor impact on its shape after deposition. 

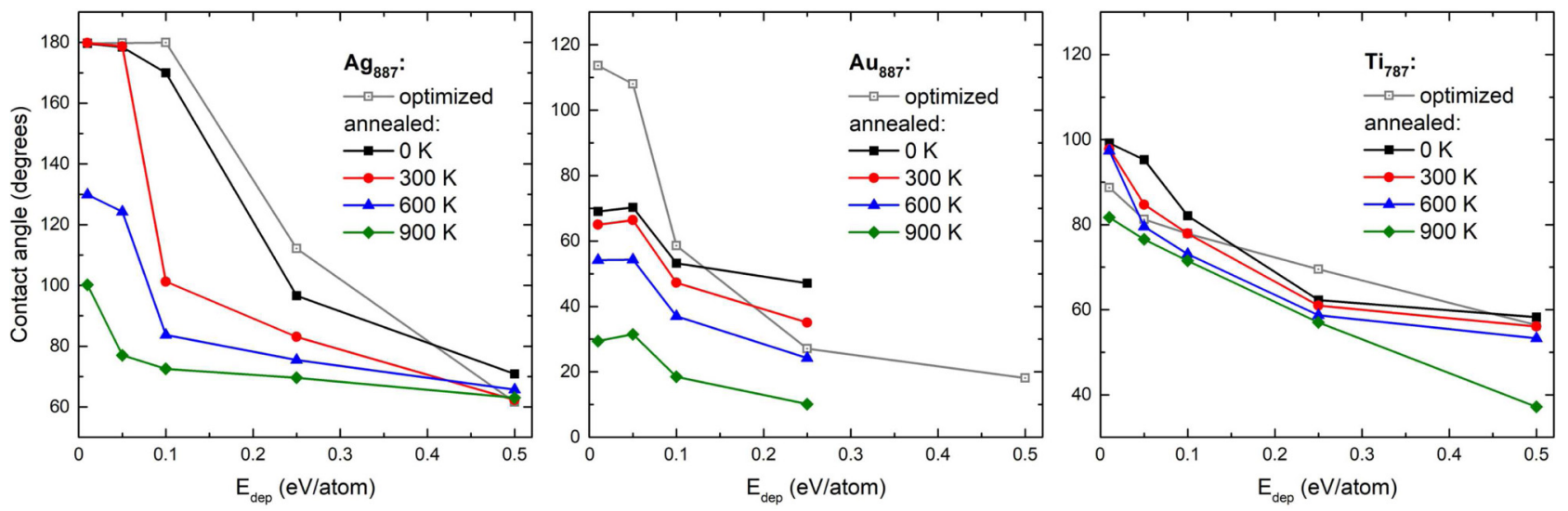

Fig. 6. Contact angle $\theta$ as a function of deposition energy for the $\mathrm{Ag}_{887}, \mathrm{Au}_{887}$ and $\mathrm{Ti}_{787}$ clusters, either optimized (open symbols) or annealed and then equilibrated at a given temperature (closed symbols).
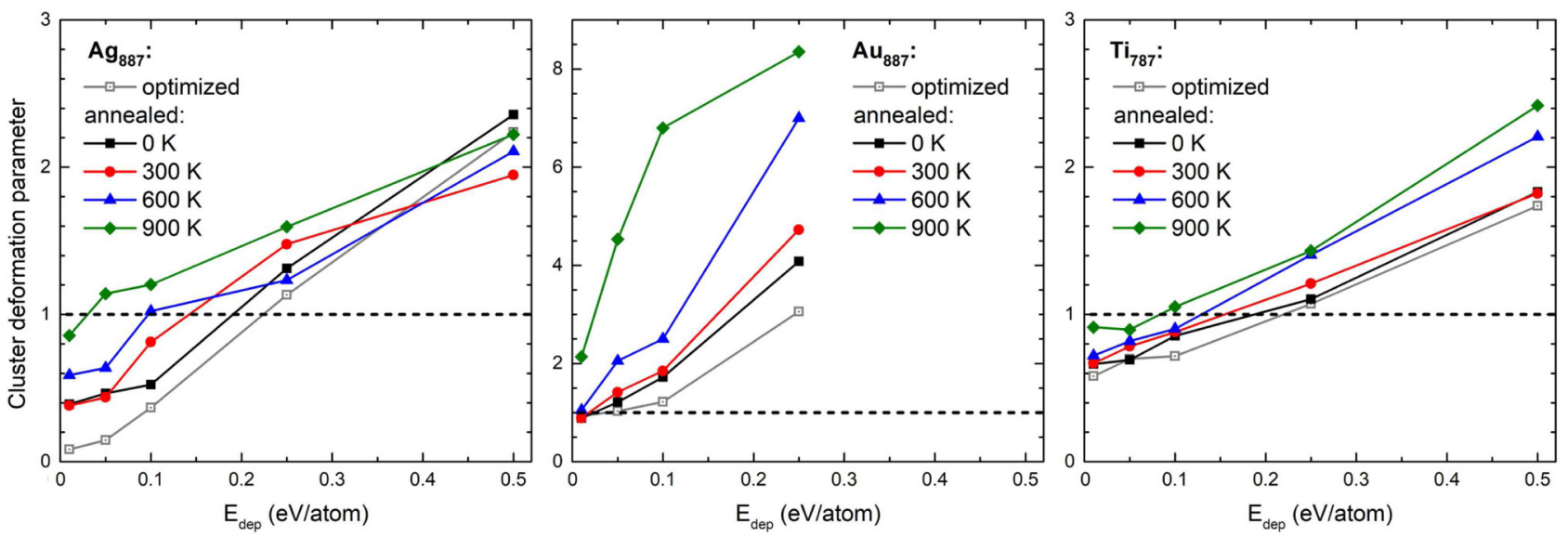

Fig. 7. The cluster deformation parameter $\delta$ as a function of deposition energy for the $\mathrm{Ag}_{887}, \mathrm{Au}_{887}$ and Ti ${ }_{787}$ clusters, either optimized (open symbols) or annealed and then equilibrated at a given temperature (closed symbols).

A series of experiments [19-21] studied the formation of fractal-like silver nanostructures on graphite after softlanding of nanometer-sized silver clusters. In these works, clusters containing from about 150 to 900 atoms were deposited at the energy of $0.05 \mathrm{eV} /$ atom. As follows from our analysis, the $\mathrm{Ag}_{887}$ cluster deposited at energies below $0.1 \mathrm{eV} /$ atom acquires an ellipsoidal shape upon deposition and its contact angle is close to 180 degrees. One may expect that clusters having such a large contact angle will be more mobile as compared to the clusters with much smaller contact angles. The question of how the shape and the contact angle of the clusters affect their diffusion properties is an interesting question which, however, goes beyond the scope of the present study.

Finally, let us analyze how pre-equilibration of the clusters at different temperatures affects their stability and shape upon deposition. Figures 6 and 7 show the contact angle and the deformation parameter of the clusters after they were given an initial temperature of $300 \mathrm{~K}, 600 \mathrm{~K}$ and $900 \mathrm{~K}$. The latter value is just above the calculated melting temperature of $\mathrm{Ag}_{887}$ and $\mathrm{Au}_{887}$ but about 100 degrees less than the melting temperature of $\mathrm{Ti}_{787}$. Therefore, the silver and gold clusters equilibrated at $900 \mathrm{~K}$ are deposited as liquid droplets while the titanium cluster has a molten surface but its core is still, at least partly, in the solid phase. As one may expect, deposition of the clusters at elevated temperature leads to a significant decrease of the contact angle and an increased contact area at low deposition energies. The contact angle for $\mathrm{Ag}_{887}$ saturates with an increase of $E_{\mathrm{dep}}$ at the value of about 60 degrees, independent on the cluster initial temperature. In contrast, the shape of $\mathrm{Au}_{887}$ depends strongly on the amount of internal energy stored in the cluster. While the profile of the $\theta\left(E_{\mathrm{dep}}\right)$ dependence does not change when the initial temperature is increased from $0 \mathrm{~K}$ to $900 \mathrm{~K}$, a decrease of the contact angle is evident.

Further insights into the stability of clusters on the graphite surface can be drawn from the analysis of radial distribution function (RDF). Figure 8 shows an exemplary $\mathrm{RDF}$ for the $\mathrm{Au}_{887}$ cluster, pre-equilibrated at $300 \mathrm{~K}$ prior deposition, at different time instances $t$ after the collision. Mechanical stress induced by the collision causes the formation of a liquid droplet within the first $10 \mathrm{ps}$ after the collision (solid blue curve). Over the next 40 ps the droplet re-crystallizes into a solid structure whose RDF resembles the initial one (dashed yellow curve). This structure 


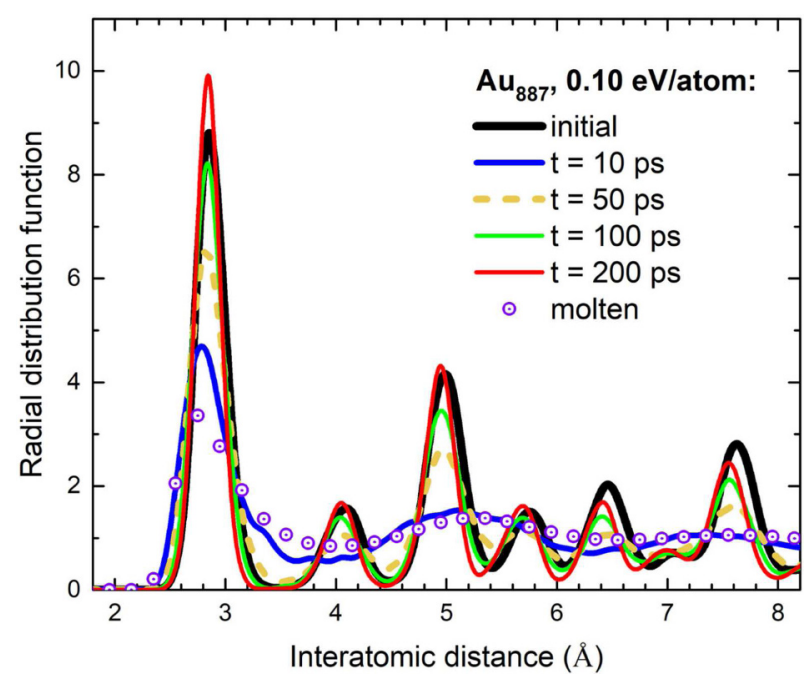

Fig. 8. Radial distribution function for the $\mathrm{Au}_{887}$ cluster, preequilibrated at $300 \mathrm{~K}$, at different time instances $t$ after the collision. An RDF for the molten state was obtained from a simulation of melting of a free $\mathrm{Au}_{887}$ cluster.

remains stable and practically does not change in the remaining part of the simulation. These observations support the results shown in Figure 3 that the change of the internal energy of the deposited gold cluster exceeds the change of the internal energy of the cluster upon its melting.

Further analysis reveals that the clusters undergo structural transformations induced by the collision. Figure 9 compares RDFs for the annealed $\mathrm{Ag}_{887}, \mathrm{Au}_{887}$ and $\mathrm{Ti}_{787}$ clusters deposited at 0.05 and $0.25 \mathrm{eV} /$ atom. The RDFs are calculated at the end of each 250 -ps long trajectory. Lattice structure of the gold cluster deposited at $0.25 \mathrm{eV} /$ atom becomes compressed as compared to the initial structure (see middle panel). This is evident from the fact that all the peaks in the RDF are strongly suppressed and shifted towards smaller interatomic distances. The silver cluster deposited at both 0.05 and $0.25 \mathrm{eV} /$ atom maintains the short-range order (see left panel). However, at $E_{\text {dep }}=0.05 \mathrm{eV} /$ atom the long-range order is lost as the peaks in the interatomic distance range of 6-8 $\AA$ are merged into a broader and more uniform distribution. In this case, the cluster does not experience the melting phase transition and subsequent recrystallization (see Fig. 3) but forms an amorphous-like structure without long-range order, which is stable on the simulation timescale of $250 \mathrm{ps}$. Amorphization of the $\mathrm{Ag}_{887}$ cluster deposited at $0.05 \mathrm{eV} /$ atom can also be seen in Figure 2. In contrast, lattice structure of the silver cluster deposited at $E_{\text {dep }}=0.25 \mathrm{eV} /$ atom resembles, to a large extent, the initial lattice structure. The only important difference is the formation of a shoulder at the interatomic distance of about $5.5 \AA$ and a shift of another peak centered at $7.1 \AA$ to $6.9 \AA$ (see green arrows). This is an indication of an increased ratio of hcp lattice packing in the deposited cluster structure. RDFs for the $\mathrm{Ti}_{787}$ cluster (right panel) illustrate the opposite phenomenon, namely a shift of the peaks centered at $5.5 \AA$ and $6.9 \AA$ towards larger inter- atomic distances, that is indicative for an increased ratio of fcc lattice in the final cluster structure.

\section{Conclusion}

The deposition of metal clusters made of three different elements - silver, gold and titanium - on a graphite substrate was studied by means of molecular dynamics simulations using the MBN Explorer and MBN Studio software packages. The clusters had the diameter of $3 \mathrm{~nm}$ and contained $N_{\mathrm{Ag}}=N_{\mathrm{Au}}=887$ and $N_{\mathrm{Ti}}=787$ atoms. We focused on deposition energies in the range $0.001-5.0 \mathrm{eV} /$ atom, which corresponds to the soft-landing regime utilized in experiments. The shape, contact angle, contact radius and height of the deposited clusters were systematically analyzed for different deposition energies $E_{\text {dep }}$ and temperature regimes. Parameters for the interaction between metal atoms and a carbon surface were determined from ab initio calculations employing the secondorder Møller-Plesset (MP2) perturbation theory.

We found that the shape and stability of deposited clusters depend strongly on the element type. At low deposition energies, the $\mathrm{Ag}_{887}$ cluster acquires a quasi-ellipsoid shape while the gold and titanium clusters rearrange upon collision into truncated oblate and prolate spheroids. Such behavior for the $\mathrm{Ag}_{887}$ deposited at $E_{\text {dep }} \lesssim 0.1 \mathrm{eV} /$ atom arises due to collision-induced plastic deformation of the cluster. A larger increase of the internal energy of the $\mathrm{Au}_{887}$ and $\mathrm{Ti}_{787}$ clusters initiates the melting phase transition in the clusters followed by their recrystallization. These processes lead to the formation of the droplet-like shapes of the clusters in a form of truncated spheroids. Both silver and gold clusters flatten over the surface and eventually disintegrate as the deposition energy increases up to $0.75-1.0 \mathrm{eV} /$ atom, while the titanium cluster fragments at about four times higher energy.

The positions of cluster surface atoms obtained from MD simulations were fitted with a surface equation. From this fit, the contact angle, contact radius and height of the clusters were determined as functions of deposition energy. We found that the contact angle for the noble metal and titanium clusters evolves differently with an increase of deposition energy. The contact angle for the silver cluster does not vary significantly at low deposition energies up to $0.1 \mathrm{eV} /$ atom, while it rapidly decreases by about 70 degrees at higher energies. The rapid decrease of the contact angle is attributed to the melting and follow-up recrystallization of the cluster in the course of the deposition. For the gold cluster, the angle drops rapidly from $130^{\circ}$ down to $20^{\circ}$ at $E_{\text {dep }}=0.5 \mathrm{eV} /$ atom. The contact angle for the titanium cluster decreases gradually from $100^{\circ}$ to $45^{\circ}$ in the range of deposition energies considered.

We also analyzed how the initial structure (optimized vs. annealed geometries) and internal energy of the clusters affect the shape and stability of the deposited clusters. Pre-equilibration of clusters at elevated temperatures up to $900 \mathrm{~K}$ results in a significant decrease of the contact angle, although this trend is different for the silver, gold and titanium clusters. The shape of $\mathrm{Ti}_{787}$ is rather 


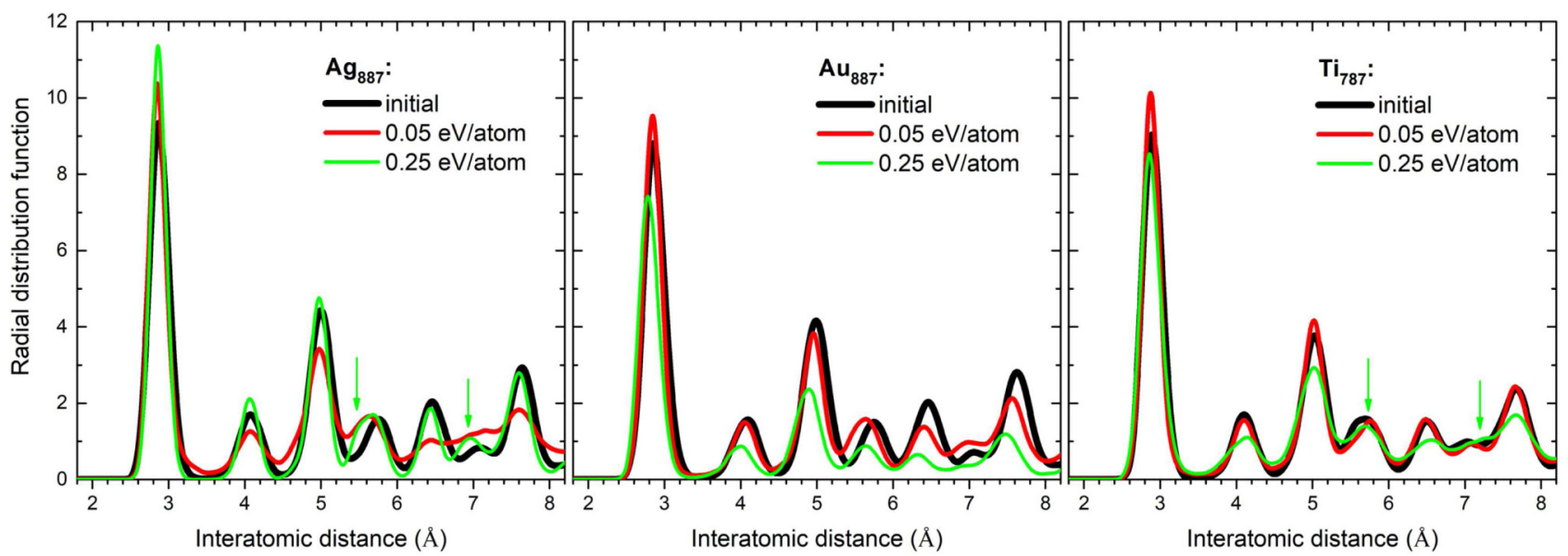

Fig. 9. Radial distribution function for the $\mathrm{Ag}_{887}, \mathrm{Au}_{887}$ and $\mathrm{Ti}_{787}$ clusters, pre-equilibrated at $300 \mathrm{~K}$, deposited at energies of 0.05 and $0.25 \mathrm{eV} /$ atom. Arrows show the appearance of fine structures in the RDFs that are indicative for fcc $\rightarrow$ hcp (in the case of silver) and hcp $\rightarrow$ fcc (in the case of titanium) structural transformations.

stable at cluster temperatures up to $900 \mathrm{~K}$ and at deposition energies up to $0.25 \mathrm{eV} /$ atom, whereas the shape of thermally excited $\mathrm{Ag}_{887}$ and $\mathrm{Au}_{887}$ clusters changes significantly even at low deposition energies.

We have considered three exemplar case studies of metal clusters interacting with graphite via different mechanisms: (i) physisorption of a silver cluster on graphite occurs due to the weak van der Waals interaction between silver and carbon atoms; (ii) the interaction of a gold cluster with graphite is mediated by van der Waals forces with a small onset of covalent bonding between gold and carbon atoms; and (iii) a much stronger titanium-graphite interaction arises due to a $p-d$ hybridization occurring between atomic orbitals of carbon and titanium atoms. Results of this study may be generalized to clusters made of other elements, which interact with a substrate by one of the above described mechanisms. For instance, earlier DFT calculations of various metal-graphene interfaces [64] showed that $\mathrm{Co}, \mathrm{Ni}$ and Pd atoms significantly disturb electronic structure of graphene, whereas $\mathrm{Al}, \mathrm{Cu}$ and $\mathrm{Pt}$ atoms interact with carbon atoms through weak van der Waals forces and do not alter electronic structure of graphene.

This work was supported in part by Deutsche Forschungsgemeinschaft (Project no. 415716638) and by the European Union's Horizon 2020 research and innovation programme the Radio-NP project (GA 794733) within the H2020-MSCAIF-2017 call and the RADON project (GA 872494) within the H2020-MSCA-RISE-2019 call. The possibility to perform calculations at the Goethe-HLR cluster of the Frankfurt Center for Scientific Computing and at the DeiC National HPC Center (University of Southern Denmark, Odense) is gratefully acknowledged.

\section{Author contribution statement}

AVV and AVS conceived the study. AVV and YE performed the simulations, analyzed the results and drafted the manuscript under the guidance of AVS. All authors participated in the discussion of the results and contributed to the revision of the manuscript.

Publisher's Note The EPJ Publishers remain neutral with regard to jurisdictional claims in published maps and institutional affiliations.

\section{References}

1. P. Jensen, Rev. Mod. Phys. 71, 1695 (1999)

2. K.-H. Meiwes-Broer, Metal Clusters at Surfaces: Structure, Quantum Properties, Physical Chemistry (Springer Verlag, Berlin, Heidelberg, 2000)

3. J.-P. Connerade, A.V. Solov'yov, Latest Advances in Atomic Cluster Collisions: Structure and Dynamics from the Nuclear to the Biological Scale (Imperial College Press, 2008)

4. K. Bromann, C. Félix, H. Brune, W. Harbich, R. Monot, J. Buttet, K. Kern, Science 274, 956 (1996)

5. S.J. Carroll, P. Weibel, B. von Issendorff, L. Kuipers, R.E. Palmer, J. Phys.: Condens. Matter 8, L617 (1996)

6. M. Hillenkamp, S.-S. Jester, M.M. Kappes, J. Chem. Phys. 116, 6764 (2002)

7. A. Kaplan, A. Bekkerman, B. Tsipinyuk, E. Kolodney, Phys. Rev. B 79, 233405 (2009)

8. H.-P. Cheng, U. Landman, J. Phys. Chem. 98, 3527 (1994)

9. P. Thaler, A. Volk, M. Ratschek, M. Koch, W.E. Ernst, J. Chem. Phys. 140, 044326 (2014)

10. V. Bernstein, E. Kolodney, J. Chem. Phys. 145, 044303 (2016)

11. V.N. Popok, I. Barke, E.E.B. Campbell, K.-H. MeiwesBroer, Surf. Sci. Rep. 66, 347 (2011)

12. S. Pratontep, P. Preece, C. Xirouchaki, R.E. Palmer, C.F. Sanz-Navarro, S.D. Kenny, R. Smith, Phys. Rev. Lett. 90, 055503 (2003)

13. R. Smith, R.P. Webb, Proc. R. Soc. London, Ser. A 441, 495 (1993)

14. S.R. Plant, L. Cao, F. Yin, Z.W. Wang, R.E. Palmer, Nanoscale 6, 1258 (2014) 
15. Z.W. Wang, R.E. Palmer, Phys. Rev. Lett. 108, 245502 (2012)

16. L. Bardotti, P. Jensen, A. Hoareau, M. Treilleux, B. Cabaud, Phys. Rev. Lett. 74, 4694 (1995)

17. R. Alayan, L. Arnaud, M. Broyer, E. Cottancin, J. Lermé, S. Marhaba, J.L. Vialle, M. Pellarin, Phys. Rev. B 76, 075424 (2007)

18. W.D. Luedtke, U. Landman, Phys. Rev. Lett. 82, 3835 (1999)

19. C. Bréchignac, P. Cahuzac, F. Carlier, C. Colliex, J. Leroux, A. Masson, B. Yoon, U. Landman, Phys. Rev. Lett. 88, 196103 (2002)

20. A. Lando, N. Kébaïli, Ph. Cahuzac, C. Colliex, M. Couillard, A. Masson, M. Schmidt, C. Bréchignac, Eur. Phys. J. D 43, 151 (2007)

21. I.A. Solov'yov, A.V. Solov'yov, N. Kébaili, A. Masson, C. Bréchignac, Phys. Status Solidi B 251, 609 (2014)

22. R.E. Palmer, S. Pratontep, H.-G. Boyen, Nat. Mater. 2, 443 (2003)

23. S. Vajda, M.G. White, ACS Catal. 5, 7152 (2015)

24. P. Milani, M. Sowwan, Cluster Beam Deposition of Functional Nanomaterials and Devices, in Frontiers of Nanoscience (Elsevier, 2020), vol. 15

25. D.N. Poenaru, R.A. Gherghescu, A.V. Solov'yov, W. Greiner, Europhys. Lett. 79, 63001 (2007)

26. D.N. Poenaru, R.A. Gherghescu, I.H. Plonski, A.V. Solov'yov, W. Greiner, Eur. Phys. J. D 47, 379 (2008)

27. V.V. Semenikhina, A.G. Lyalin, A.V. Solov'yov, W. Greiner, J. Exp. Theor. Phys. 106, 678 (2008)

28. A.V. Prydatko, L.A. Belyaeva, L. Jiang, L.M.C. Lima, G.F. Schneider, Nat. Commun. 9, 4185 (2018)

29. M. Lundgren, N.L. Allan, T. Cosgrove, N. George, Languir 18, $10462(2002)$

30. F. Song, L. Ma, J. Fan, Q. Chen, G. Lei, B.Q. Li, Phys. Chem. Chem. Phys. 20, 11987 (2018)

31. P.V. Kashtanov, R. Hippler, B.M. Smirnov, S.R. Bhattacharyya, J. Exp. Theor. Phys. 110, 521 (2010)

32. Z. Chen, J.B. Lee, ACS Appl. Mater. Interfaces 11, 35488 (2019)

33. M. Couillard, S. Pratontep, R.E. Palmer, Appl. Phys. Lett. 82, 2595 (2003)

34. I.A. Solov'yov, A.V. Yakubovich, P.V. Nikolaev, I. Volkovets, A.V. Solov'yov, J. Comput. Chem. 33, 2412 (2012)

35. G.B. Sushko, I.A. Solov'yov, A.V. Solov'yov, J. Mol. Graphics Modell. 88, 247 (2019)

36. I.A. Solov'yov, G.B. Sushko, A.V. Solov'yov, $M B N$ Explorer Users' Guide, ver. 3.0 (MesoBioNano Science Publishing, Frankfurt am Main, 2017)

37. R.P. Gupta, Phys. Rev. B 23, 6265 (1981)

38. F. Cleri, V. Rosato, Phys. Rev. B 48, 22 (1993)
39. T. Ellaby, J. Aarons, A. Varambhia, L. Jones, P. Nellist, D. Ozkaya, M. Sarwar, D. Thompsett, C.-K. Skylaris, J. Phys.: Condens. Matter 30, 155301 (2018)

40. D.W. Brenner, Phys. Rev. B 42, 9458 (1990)

41. J. Geng, I.A. Solov'yov, W. Zhou, A.V. Solov'yov, B.F.G. Johnson, J. Phys. Chem. C 113, 6390 (2009)

42. J.H. Ryu, H.Y. Kim, D.H. Kim, D.H. Seo, H.M. Lee, J. Phys. Chem. C 114, 2022 (2010)

43. M. Neek-Amal, R. Asgari, M.R. Rahimi Tabar, Nanotechnology 20, 135602 (2009)

44. Ş. Erkoç, Annu. Rev. Comput. Phys. IX, 1 (2001)

45. A.V. Verkhovtsev, S. Schramm, A.V. Solov'yov, Eur. Phys. J. D 68, 246 (2014)

46. A.Y. Galashev, K.P. Katin, M.M. Maslov, Phys. Lett. A 383, 252 (2019)

47. P.V.C. Medeiros, G.K. Gueorguiev, S. Stafström, Phys. Rev. B 85, 205423 (2012)

48. J. Björk, S. Stafström, F. Hanke, J. Am. Chem. Soc. 133, 14884 (2011)

49. O.I. Obolensky, V.V. Semenikhina, A.V. Solov'yov, W. Greiner, Int. J. Quantum Chem 107, 1335 (2007)

50. S. Grimme, WIREs Comput. Molec. Sci. 1, 211 (2011)

51. M. Amft, S. Lebègue, O. Eriksson, N.V. Skorodumova, J. Phys.: Condens. Matter 23, 395001 (2011)

52. J.-P. Jalkanen, M. Halonen, D. Fernández-Torre, K. Laasonen, L. Halonen, J. Phys. Chem. A 111, 12317 (2007)

53. M.J. Frisch et al., Gaussian 09, Revision D.01 (Gaussian, Inc., Wallingford CT, 2013)

54. L. Chen, J. Luo, Q. Wang, L. Xiong, H. Gong, J. Phys.: Condens. Matter 32, 145001 (2020)

55. A.L. Hsu, R.J. Koch, M.T. Ong, W. Fang, M. Hofmann, K.K. Kim, T. Seyller, M.S. Dresselhaus, E.J. Reed, J. Kong, T. Palacios, ACS Nano 8, 7704 (2014)

56. I. Katakuse, T. Ichihara, Y. Fujita, T. Matsuo, T. Sakurai, H. Matsuda, Int. J. Mass Spectrom. Ion Processes 67, 229 (1985)

57. H. Häkkinen, Adv. Phys. X, 1, 467 (2016)

58. P.-G. Reinhard, E. Suraud, Introduction to Cluster Dynamics (Wiley-VCH Verlag, Weinheim, 2004)

59. A. Awasthi, S.C. Hendy, P. Zoontjens, S.A. Brown, Phys. Rev. Lett. 97, 186103 (2006)

60. N. Giovambattista, P.G. Debenedetti, P.J. Rossky, J. Phys. Chem. B 111, 9581 (2007)

61. J. Škvára, J. Škvor, I. Nezbeda, Molec. Simul. 44, 190 (2018)

62. N. Lidgi-Guigui, P. Mulheran, R.E. Palmer, Appl. Phys. Lett. 93, 123107 (2008)

63. F. Claeyssens, S. Pratontep, C. Xirouchaki, R.E. Palmer, Nanotechnology 17, 805 (2006)

64. G. Giovannetti, P.A. Khomyakov, G. Brocks, V.M. Karpan, J. van den Brink, P.J. Kelly, Phys. Rev. Lett. 101, 026803 (2008) 